\title{
Predicting the Behaviour of Near-Critical and Supercritical Alcohols at Microwave Frequencies: Validation of Molecular Dynamic Simulations as a Tool that can Substitute for Measurements under Extreme Experimental Conditions
}

David Robinson, ${ }^{1}$ Derek J. Irvine, ${ }^{2}$ John P. Robinson, ${ }^{2}$ Edward H. Lester,${ }^{2}$ Samuel W. Kingman, ${ }^{2}$ and Georgios Dimitrakis ${ }^{2, *}$

*Corresponding author: E-mail georgios.dimitrakis@nottingham.ac.uk

${ }^{1}$ Department of Chemistry and Forensics, School of Science and Technology, Nottingham Trent University, Clifton Lane, Nottingham, NG11 8NS, United Kingdom.

${ }^{2}$ Department of Chemical and Environmental Engineering, Faculty of Engineering, University of Nottingham, University Park, Nottingham, NG7 2RD, United Kingdom.

Abstract: Equilibrium and non-equilibrium molecular dynamic simulations, predicting the dielectric properties of near-critical and supercritical methanol and ethanol at microwave frequencies have been carried out. The autocorrelation functions of the dielectric relaxation, show dependency on the slow component at the near-critical region for both alcohols. At the supercritical region, two competing relaxation mechanisms are observed, related to the large breakdown of the hydrogen-bonding network and the degree of clustering between the molecules. This approach closely matches experimental data at microwave frequencies and identical temperature and pressure conditions, validating the predictions of how the molecular structure and dynamics manifest themselves into the complex permittivity and dielectric relaxation behaviour. Thus, introducing a modelling-based solution to deliver accurate dielectric property values for materials 
at supercritical conditions for "a priori" screening of solvents, whilst removing the need to overcome engineering and safety challenges associated with the development of experimental equipment to practically generate such data.

Keywords: Complex permittivity, dielectric relaxation times, molecular dynamics simulations, Drude polarisable force-field.

\section{Introduction}

Recent years have witnessed an increased interest and shift towards more sustainable "green" processes in the chemical and engineering communities [1-3]. Supercritical fluids are prime candidates for use as solvents in such chemistries as the majority of them are compatible with the environment and generally possess enhanced transport properties for reactions [4].

Knowledge of the dielectric properties of supercritical fluids is important in determining the effect that the solvent has on the equilibrium of the reactions occurring at these conditions [5]. Recent experimental work in the field of green chemistry has also highlighted the potential benefits of combining the chemical properties that supercritical and near critical fluids can exhibit with the excellent heating potential offered by microwave energy during chemical reactions [6-7]. Microwave energy is capable of heating a material volumetrically and rapidly [8]. This in conjunction with the excellent microwave absorption properties of alcohols and water has led to novel research in the development of equipment capable of producing supercritical or near critical fluids with the use of microwaves for chemical reactions [9], or carrying out reactions with microwave energy at high pressures [10]. The optimum design and development of such equipment requires intimate knowledge of the dielectric properties of the materials subjected to microwave heating [8]. The term "dielectric properties" typically refers to the knowledge of the complex permittivity $\varepsilon^{*}$ of a medium. Complex permittivity consists of two parts, a real part which 
is termed the dielectric constant, $\varepsilon^{\prime}$, and an imaginary part called dielectric loss, $\varepsilon^{\prime \prime}$ [11]. The first is related to the polarisation and energy stored within a material in the presence of an external electric or electromagnetic field, and the latter correlated to energy lost in the material [12]. Dielectric properties are essential in understanding the fundamental properties of polar fluids [11] and their response to microwave fields. This is could be particularly important since a substantial number of solvents used in supercritical chemistry (water, alcohols etc.) are extremely polar under ambient conditions.

Consequently, a significant range of dielectric property measurement techniques have been established by the scientific community to generate such data [13-15]. However, in practice these measurements are very difficult to conduct under extreme experimental conditions, such as high temperature and pressure. In particular, the integration of the sensitive electronic equipment required to make the measurement into the experimental apparatus has proved challenging. Also experimentation at high pressures and temperatures constitutes an inherent safety hazard with risks that needs to be carefully mitigated. Typically all experimental equipment used for that purpose need to be enclosed in a bullet proof cage as a potential explosion can be catastrophic. As a result, there are very few reports in the literature relating to these types of measurements [16-18], despite the potential benefits that such knowledge of the dielectric properties of systems at the near-critical or supercritical state might have. Therefore, an alternative method that overcomes the difficulties associated with the measurements in these challenging environments would potentially be very valuable. Dielectric property measurements can be directly linked to the molecular orientation of a specific entity within the electric field. Thus, validated molecular dynamics (MD) simulations are potentially an extremely useful method for understanding, interpreting and even predicting experimental data relating to these processes, with atomistic topological and sub-picosecond 
temporal resolution, possibly alleviating the need of using onerous experimental methodologies to generate such data.

Studies of different molecules under ambient conditions have utilized molecular dynamics (MD) in order to calculate the molecular dielectric response. Gnanasekaran and Leitner have investigated the dielectric response of the photoactive yellow protein [19] following an $S_{1} \leftarrow S_{0}$ electronic transition, using correlation functions to estimate the dielectric response. Fourier transforms of the correlation function indicated the excited-state interactions between amino acid residues, in agreement with experiment. The dielectric relaxation properties of ethanol at room temperature have also been studied with MD [20]. The dielectric properties of supercritical water were reported by Guàrdia and Martí [21]. In this study, the authors found that, as water reached the supercritical phase, rotational correlation times of the water molecules became important as the hydrogen bond network broke down, leading to a more "gas-phase" behaviour. Additionally, several studies on non-critical mixtures of methanol, ethanol, water and other alcohols have been successfully studied using MD simulations [22-25].

In the present study, we consider the dielectric relaxation of near-critical and supercritical methanol and ethanol using MD with a polarisable force-field and comparing with our previously obtained experimental dielectric measurement data. The choice of ethanol and methanol was purely based on the fact that both are extremely common solvents, having a relative low environmental impact. This provides us with a computational approach which is able to predict the dielectric properties of near- and super-critical fluids, which otherwise are very difficult to measure experimentally, allowing an effective method of "screening" different molecular compositions before undertaking dedicated experimental measurements.

\section{Computational Details}


The all-atom force-field for methanol and ethanol were employed [26], within the Drude polarisable force-field framework [27-29]. The initial structure(s) consisted of 3200 alcohol molecules, using cubic symmetry. Initially, 250 steps of steepest-descent minimization were used to remove any bad contacts, followed by a further 2000 steps of adapted basis Newton-Raphson (ABNR) minimization. Equilibration dynamics were performed initially using a $1 \mathrm{fs}$ time step for 300 ps with a non-bond cutoff of $16 \AA$, with the switching function starting at $10 \AA$ and eliminating all pair contributions at $12 \AA$. Long-range electrostatics were treated using the Particle Mesh Ewald (PME) algorithm, using a $6^{\text {th }}$-order spline interpolation. Langevin temperature control was used, with damping of $10 \mathrm{ps}^{-1}$. Periodic boundary conditions were used, using cubic symmetry. The SHAKE/Roll and RATTLE/Roll algorithms were employed to constrain covalent bonds to hydrogen. A dual Langevin thermostat was used, such that the Drude particles were kept at $272^{\circ} \mathrm{C}(1 \mathrm{~K})$, while the real atoms were kept close to $100^{\circ} \mathrm{C}, 120^{\circ} \mathrm{C}, 135^{\circ} \mathrm{C}, 150^{\circ} \mathrm{C}, 165^{\circ} \mathrm{C}, 180^{\circ} \mathrm{C}$, $200^{\circ} \mathrm{C}$ and $250^{\circ} \mathrm{C}$. The canonical ensemble (NVT) was chosen in order to maintain the correct densities required for direct comparison with experimental data of near-critical and supercritical methanol and ethanol (densities used are given in Table 1, along with experimental densities [30,31]). Preliminary isobaric-isothermal ensemble (NPT) simulations at the experimental pressures were also performed; where experimental densities were not available, the calculated densities from these NPT simulations were used in the main NVT simulations, as they were found to agree well with the experimental densities for ethanol. Equilibration lasted for $20 \mathrm{ns,} \mathrm{while}$ production dynamics were carried out under the same conditions (without temperature rescaling) for a total of $100 \mathrm{~ns}$ per temperature simulated. Two separate simulations were performed at each temperature; one as described above, and a second, non-equilibrium simulation, with an applied oscillating electric field at a frequency of $2.45 \mathrm{GHz}$ (most commonly used for laboratory scale microwave heating experiments), and an intensity of $2000 \mathrm{Vm}^{-1}$ (it must be noted that the field is 
substantially higher to what a measuring instrument such as a vector network analyser can generate; it was chosen in order to investigate the presence of any potential perturbations at the molecular level). All of the simulations were performed with the CHARMM MD software [27]. The equilibrium simulations were used to determine the dielectric relaxation properties, while we calculated the polarisation response within the non-equilibrium simulation to confirm that the experimentally applied microwave field intensity we have previously used [18] is sufficiently low as not to alter the system characteristics, while obtaining reliable dielectric measurements.

\section{Results and Discussion}

Autocorrelation functions of the dielectric relaxation for ethanol and methanol were calculated at each of the temperatures studied from the equilibrium MD simulations. The curves were fitted to an exponential function of the form:

$F(t)=A e^{-\frac{t}{\tau_{1}}}$

where $\tau_{l}$ is the slow component of the dielectric relaxation. The calculated slow-component dielectric relaxation times are given in Table 2 . The relaxation times are in within $10 \%$ of experimental results under similar conditions [32, 33]. The exponential form of the dielectric relaxation profiles indicate that the dielectric response of the near-critical alcohols follows Debye relaxation. At $250{ }^{\circ} \mathrm{C}$, essentially identical relaxation times for both ethanol and methanol are observed. The supercritical relaxation time is discussed in more detail below.

In the analysis of the MD trajectory, the Drude particles were allowed to relax while keeping the atomic coordinates fixed to understand the effect of polarisation. The average change in the dipole moment was small, at approximately $0.2 \mathrm{D}$ (for all temperatures), in both the equilibrium and non-equilibrium simulations.

The static dielectric permittivity, $\varepsilon_{s}$, was calculated according to $\mathrm{Eq}(2)$ : 


$$
\varepsilon_{S}=1+\frac{4 \pi\left(\left\langle\overline{M^{2}}\right\rangle-\langle\bar{M}\rangle^{2}\right)}{332.072\left(3 k_{B} T V D^{2}\right)}
$$

Here, $M$ and $V$ refer to the total dipole moment and volume of the simulation box, respectively. $T$ is the temperature and $D$ is the Debye constant [33]. Using the relaxation times, $\tau$, calculated earlier and the static dielectric permittivity $\left(\varepsilon_{s}\right)$, the complex permittivities were calculated as

$$
\varepsilon^{*}(\omega)=\varepsilon_{\infty}+\frac{\varepsilon_{s}-\varepsilon_{\infty}}{1+i \omega \tau}
$$

assuming a dielectric permittivity at infinite frequency $\left(\varepsilon_{\infty}\right)$ of 1.92 [34], where $\omega$ is the angular frequency of the applied field (herein taken as $2.45 \mathrm{GHz}$ ). It should be noted that $\varepsilon_{\infty}$ is dependent on density, and as such this parameter should be varied accordingly. However, for the present work, we choose to use this particular value as it is experimentally validated at $\mathrm{T}=\left\{25-325^{\circ} \mathrm{C}\right\}$ and $\mathrm{P}=\{6.1 \mathrm{MPa}$ and $15.1 \mathrm{MPa}\}$, conditions which are close to those that we have employed in the present study, while there is not a large amount of data about $\varepsilon_{\infty}$ at different densities. Also, the degree that $\varepsilon_{\infty}$ can influence the calculated values of complex permittivity is typically small and only becomes significant at high temperatures where $\varepsilon_{s}$ decreases substantially [34].

Presented in Fig. 1 are the computed complex permittivities for methanol and ethanol from the equilibrium MD simulations, calculated using the Debye relation. These are in excellent agreement with the reported experimental data [18], with an average deviation of less than 5\%. It must be noted that the experimental results were also subject to a standard deviation (shown in Fig. 1) in the measurement of the dielectric properties, mainly due to the minor changes in the electrical length of the measurement sensor (compression of the sapphire bead in the coaxial line) imposed by the high pressure conditions during the experiment. These changes in the geometry of the sensor with pressure explain also the experimental observation that $\varepsilon^{\prime}$ is typically associated with larger 
standard deviations compared to $\varepsilon^{\prime \prime}$, since $\varepsilon^{\prime}$ is related to the phase shift of the reflection coefficient $(\Gamma)$ which is intimately linked to the length of the coaxial line. On the other hand, $\varepsilon^{\prime \prime}$ depends on the amplitude of $\Gamma$ which in general remains unaffected by the experimental conditions. The small deviations from the experimental values of $\varepsilon^{\prime}$ could be either due to the choice of density values, given the difficulty in accurately determining these experimentally or due to the experimental error introduced by the change in the effective electrical length of the sensor as described previously. The deviations for the real part of the dielectric permittivity are more significant in the supercritical region, especially for ethanol. The components of the complex permittivity $\left(\varepsilon^{\prime}\right.$ and $\left.\varepsilon^{\prime \prime}\right)$ of ethanol and methanol decrease with increasing temperature, due to the combined effect of a reduction of the static permittivity and relaxation time. Both the static permittivity and the relaxation time will decrease with temperature (which results to a relaxation frequency shift towards higher values). Since we are carrying out our measurements at $2.45 \mathrm{GHz}$ this will cause both the real part of permittivity (due to the decrease in static permittivity) and the imaginary part of permittivity (due to the displacement of the relaxation frequency further away from our measuring frequency) to decrease.

The hydrogen bond lifetimes and occupancies are given in Fig. 2 for methanol. For the current work, a hydrogen bond is considered to exist if the distance between the donor and acceptor atoms is less than $2.4 \AA$, while all angles are allowed. We allowed all angles, since there would still be an interaction even if not considered strictly a hydrogen bond. The lifetime is defined as the average time of all hydrogen bonds within the system, while occupancy is defined as the fraction of hydrogen bonds observed (for each donor) compared with the theoretical maximum number of hydrogen bonds for each donor. The hydrogen bond network breaks down with increasing temperature, with a much larger change at $250{ }^{\circ} \mathrm{C}$, when the methanol is in the supercritical state. 
Importantly, we note that this process is dependent on the temperature and independent of the applied microwave electric field intensity, by comparison of the equilibrium and non-equilibrium MD simulations. This breakdown of hydrogen bonding network is also important when considering the dielectric relaxation time. Given in Fig. 3 are the autocorrelation functions of the dielectric relaxation of supercritical methanol and ethanol $\left(250{ }^{\circ} \mathrm{C}\right)$. It is interesting to note that the curves for methanol and ethanol exhibit very similar profiles; this may be a consequence of the sampling frequency we have used in analysing the MD trajectories. The curves deviate substantially from the trend that could be expected for a process described by an exponential function, in contrast to the curves observed at lower temperatures (Fig. 4), with the initial part of the curve displaying a "backscattering-like" minimum, as has been observed experimentally [35] and is attributed to the free rotor frequency. This is due to the re-orientational correlation time of the molecule(s) to reorient around a given molecular axis and is representative of the system approaching gas-phase behaviour, as would be expected in the supercritical region. In the nonsupercritical region, the hydrogen bonding network remains strong, with hydrogen bond occupations $>0.3$, while the supercritical region sees a substantial breakdown of hydrogen bonds. However, it must be noted that during our simulation studies a complete disassociation between all the alcohol molecules at supercritical conditions was not observed. Molecules are able to retain a fraction of hydrogen bonding (approximately 0.1) whilst the hydrogen bond lifetimes are reduced to below 2 ps. In Fig. 5 are the calculated radial distribution functions for the distance between oxygen atoms of the methanol and ethanol molecules in the supercritical phase. We can observe a greater degree of clustering for methanol than ethanol in this phase. Indeed, methanol is known to form 3-5 molecule clusters around the hydroxyl hydrogen at its supercritical state [30] whereas ethanol exhibits a significant smaller degree of clustering [36]. For comparison, we also include a representative radial distribution function for methanol in the sub-critical phase $\left(150^{\circ} \mathrm{C}\right)$ and see a 
slightly different structure to that observed in the supercritical phase, although the supercritical phase still exhibits strong molecular structuring due to clustering at short separations.- We can speculate that the calculated dielectric relaxation time within the supercritical region will have significant error bars attached, as there are two processes occurring within this timeframe.

For non-equilibrium MD simulations, one should first consider the polarisation response, $\mathbf{E}_{p}$, of the system to the applied field:

$\mathbf{E}_{p}=-\frac{\mathbf{P}}{\varepsilon_{0}}$

Where, $\mathbf{P}$ is a multipole expansion given by:

$\mathbf{P}=\mathbf{P}_{1}-\nabla \cdot \mathbf{P}_{2}+\nabla \nabla: \mathbf{P}_{3}-\mathrm{K}$

The first term in Eq (5) is the dipole moment density, which can be shown to be equivalent to the charge density, while the other terms relate to higher-order (non-linear) polarisation responses [37]. By calculating the change in the polarisation response between the equilibrium MD simulation and the non-equilibrium simulation with respect to the applied field, it is possible to show that the response is first-order. In fact, for both systems studied here, at the applied field given above, the polarisation response is vanishingly small, suggesting that this field intensity is suitable to study dielectric properties experimentally without perturbing the system of interest.

Finally, we note that the preliminary NPT simulations (with pressures of $9 \mathrm{MPa}$ for methanol and $7 \mathrm{MPa}$ for ethanol) gave almost identical results to the NVT simulations at the non-critical temperatures, with densities in agreement with those chosen for the NVT simulations. However, in the supercritical region, the calculated densities deviated by more than $50 \%$ from the experimentally determined values for both alcohols; we can therefore conclude that the use of the NVT ensemble is essential to describe the supercritical conditions and dielectric properties. 


\section{Conclusions}

We have investigated the dielectric response of near-critical and supercritical methanol and ethanol by MD simulations employing a polarisable force-field. The dielectric relaxation was shown to follow an exponential decay for both methanol and ethanol, supporting a Debye-like dielectric response in the near-critical region. At the supercritical state there is still a significant fraction of hydrogen bonding present in both systems, although methanol exhibits a higher degree of clustering than ethanol. Nevertheless it is doubtful whether a single Debye relaxation can be used to describe adequately the dielectric behaviour in the supercritical region. Non-equilibrium MD simulations have revealed no dependence of the relaxation process to the intensity of an externally applied electric microwave field as high as $2000 \mathrm{~V} / \mathrm{m}$. Therefore, we can conclude that the intensity of the electric field used by typical dielectric spectroscopy techniques does not perturb the system under measurement. Calculated dielectric relaxation times are in agreement with the reported experimental values, leading to complex dielectric profiles that are in excellent agreement with the experimental data in the subcritical regime. Also the computational study has revealed that in the supercritical region the use of the NVT ensemble is essential in order to describe the dielectric properties. Through validation of the computed results with experimental data under extreme conditions, we have shown that MD simulations show qualitative and quantitative agreement in the interpretation, analysis and prediction of the dielectric properties of alcohols in the near-critical regime, with good qualitative agreement in the supercritical regime, allowing us to have a safe and effective method for the screening of molecular systems before employing very complex experimental measurements. Such data generated by MD simulations, could potentially be used at the initial design stages of experimental equipment utilising microwave energy as heating source for carrying out chemical reactions in the presence of solvents, as well as solvent screening at the near-critical or super-critical state. However, we note that accurate predictions 
using classical MD approaches are reliant on high quality force-fields being available for the molecule(s) of interest.
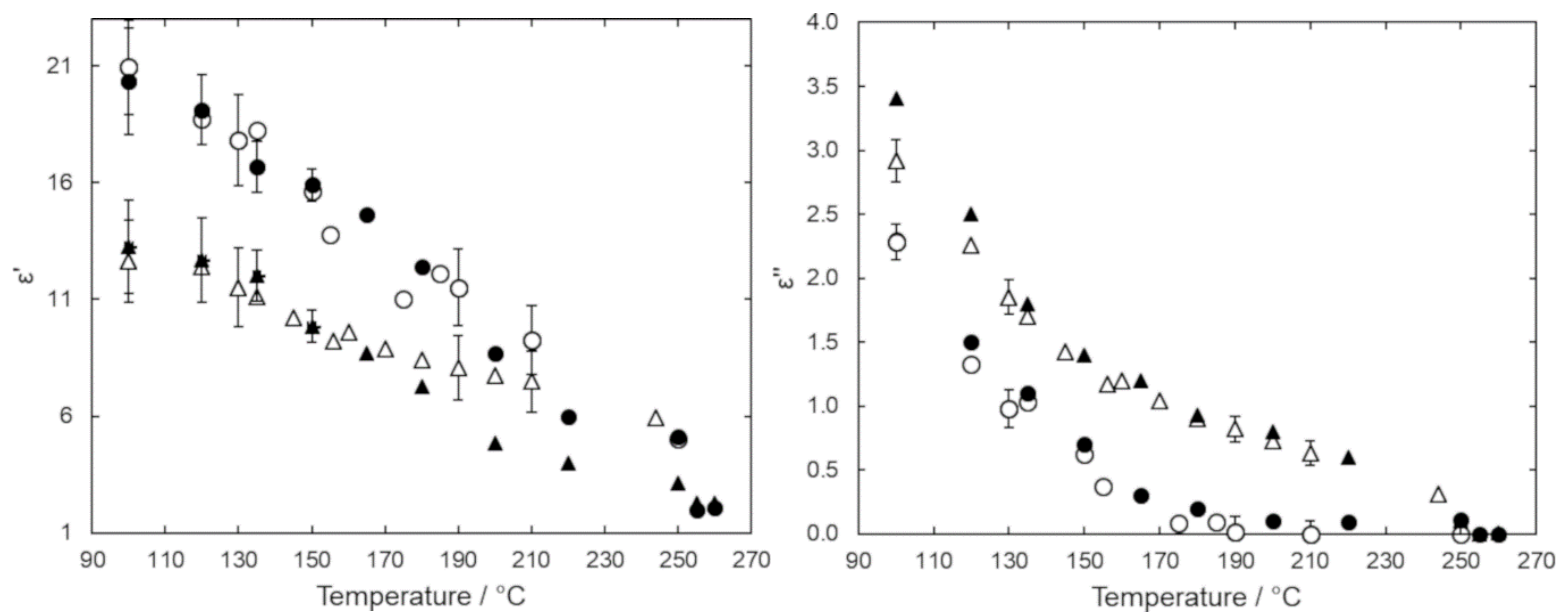

Fig. 1. Variation of the computed real and imaginary parts of the complex permittivity versus temperature for methanol (closed circles, open circles for experimental results ${ }^{18}$ ) and ethanol (closed triangles, open triangles for experimental results ${ }^{18}$ ) from the MD simulations at pressures of $9 \mathrm{MPa}$ (methanol) and $7 \mathrm{MPa}$ (ethanol) and a frequency of $2.45 \mathrm{GHz}$. 

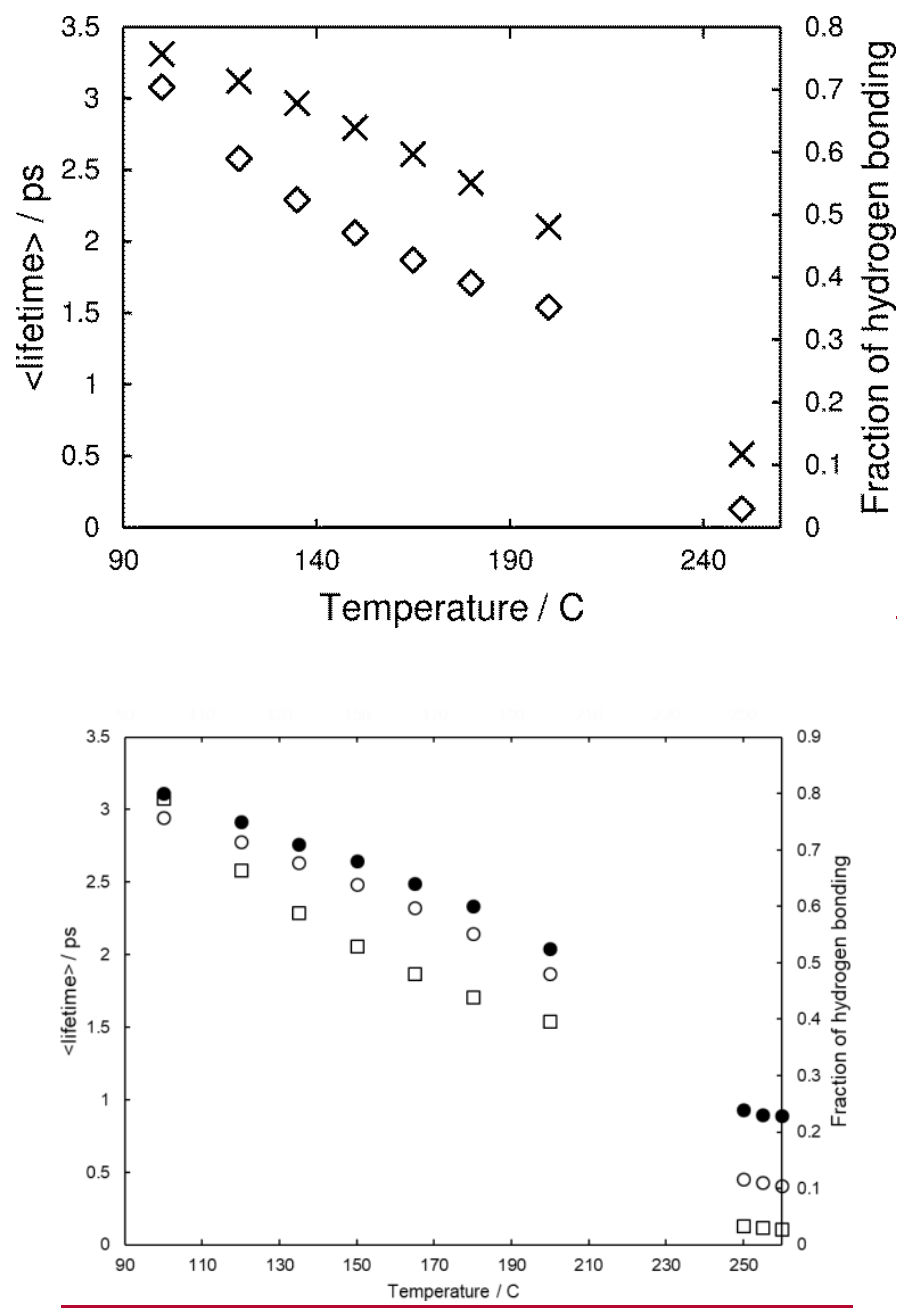

Fig. 2. Average hydrogen bond lifetime (diamendsopen squares for methanol) and fraction of hydrogen bonding (erossesopen circles for methanol; closed circles for ethanol) versus temperature for methanol. 

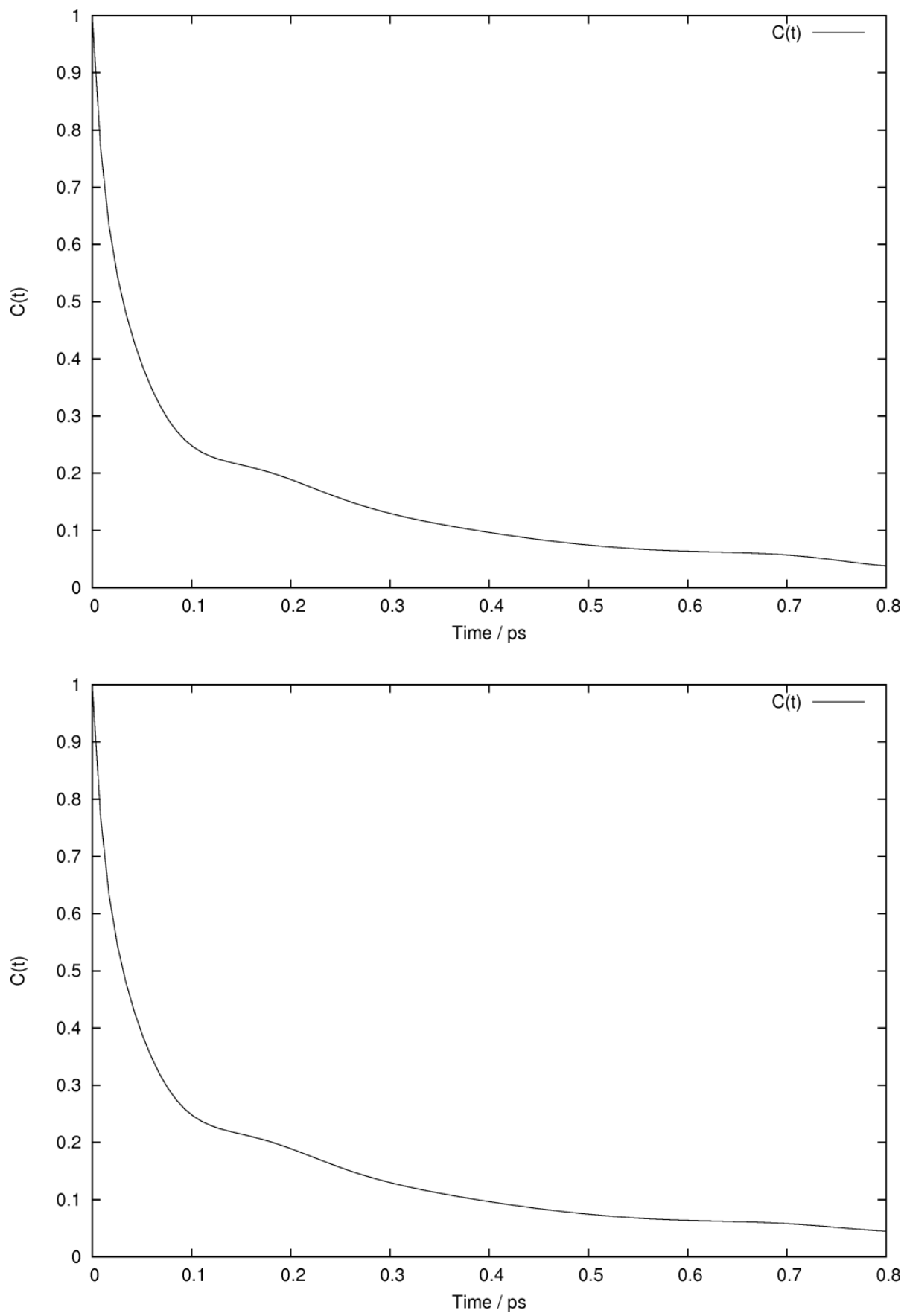

Fig. 3. Autocorrelation function for the dipole relaxation calculated for supercritical methanol (top) and ethanol (bottom) at $250^{\circ} \mathrm{C}$. 

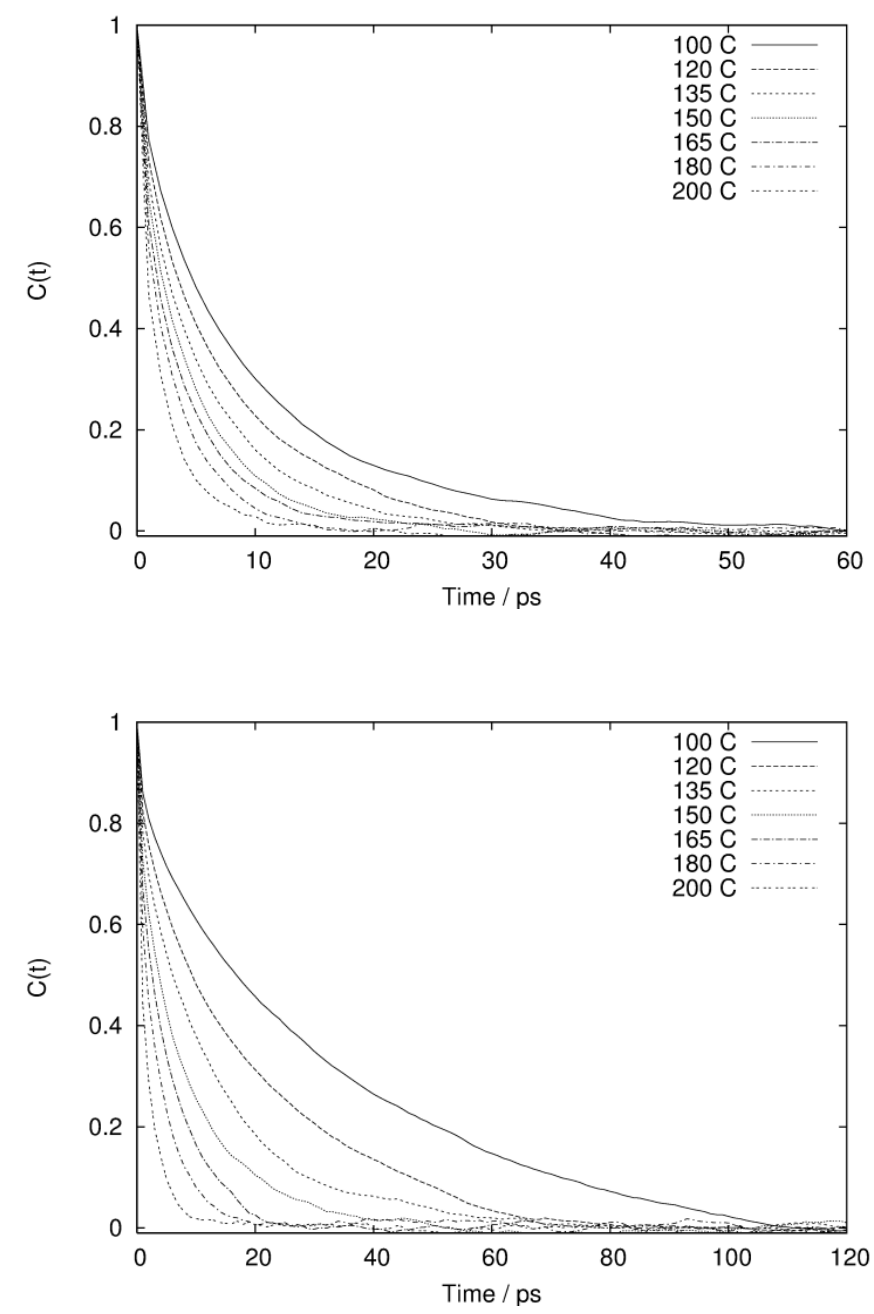

Fig. 4. Autocorrelation function showing the dipole relaxation for methanol (upper panel) and ethanol (lower panel), at various temperatures in the sub-critical region. 


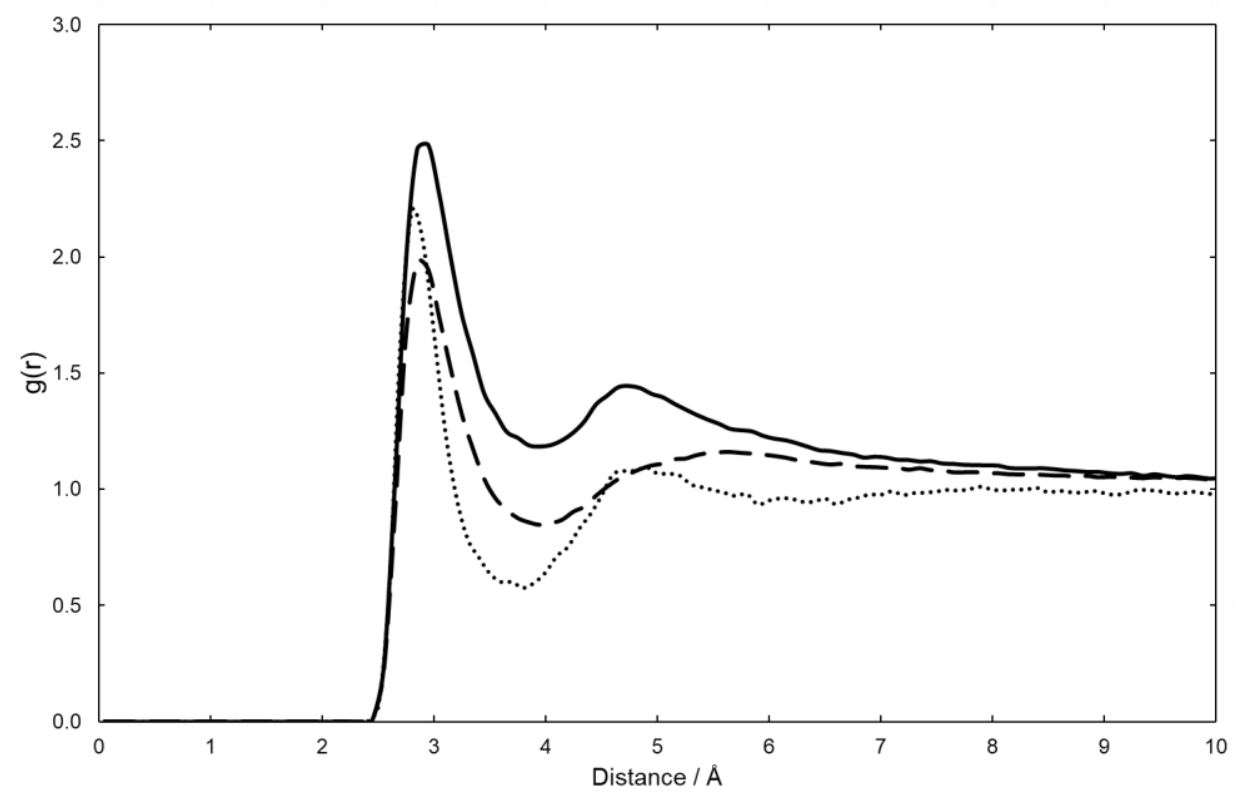

Fig. 5. Calculated radial distribution function of the $\mathrm{O}-\mathrm{O}$ distance between methanol molecules (solid line) and ethanol molecules (dashed line) in the supercritical phase. The radial distribution function of methanol at $150^{\circ} \mathrm{C}$ (dotted line) is given for comparison. 
Table 1. Densities used in the NVT simulations of the alcohols $\left(\mathrm{g} \mathrm{cm}^{-3}\right)$. Experimental densities are given in parentheses. (a) Reference [25]; (b) Reference [26].

\begin{tabular}{|c|c|c|c|c|c|c|c|c|c|c|}
\hline $\begin{array}{l}\text { Temp / } \\
{ }^{\circ} \mathrm{C}\end{array}$ & 100 & 120 & 135 & 150 & 165 & 180 & 200 & 250 & 255 & 260 \\
\hline Methanol & 0.712 & 0.686 & 0.664 & 0.639 & 0.610 & 0.576 & 0.513 & $\begin{array}{c}0.453 \\
(0.453)^{\mathrm{a}}\end{array}$ & 0.441 & 0.427 \\
\hline Ethanol & $\begin{array}{c}0.720 \\
(0.720)^{\mathrm{b}}\end{array}$ & 0.711 & 0.692 & $\begin{array}{c}0.670 \\
(0.670)^{\mathrm{b}}\end{array}$ & 0.650 & 0.626 & $\begin{array}{c}0.600 \\
(0.600)^{\mathrm{b}}\end{array}$ & $\begin{array}{c}0.229 \\
(0.229)^{\mathrm{b}}\end{array}$ & 0.209 & 0.199 \\
\hline
\end{tabular}


Table 2. Calculated dielectric relaxation times (ps). "Note that the interval for saving data to the trajectory file is $2.0 \mathrm{ps}$.

$\begin{array}{lllllllllll}\text { Temp } /{ }^{\circ} \mathrm{C} & 100 & 120 & 135 & 150 & 165 & 180 & 200 & 250 & 255^{*} & 260^{*}\end{array}$

\begin{tabular}{lcccccccccc}
\hline Methanol & 12.0 & 8.7 & 6.9 & 5.3 & 5.0 & 3.7 & 3.1 & 2.0 & 2.0 & 2.0 \\
Ethanol & 33.2 & 21.4 & 14.8 & 10.4 & 6.5 & 4.7 & 3.0 & 2.0 & 2.0 & 2.0
\end{tabular}




\section{Acknowledgments}

We would like to acknowledge the University of Nottingham HPC service for provision of time on the Minerva High Performance Computing cluster. This research did not receive any specific grant from funding agencies in the public, commercial, or not-for-profit sectors.

\section{References}

[1] P. T. Anastas, J. C. Warner. Green Chemistry: Theory and Practice, (New York: Oxford University Press), 1998.

[2] P. T. Anastas, J. B. Zimmerman. Design Through the 12 Principles of Green Engineering. Environmental Science \& Technology, 37 (2003) 94a-101a.

[3] P. T. Anastas, Perspective on Green Chemistry: The most challenging synthetic transformation, Tetrahedron 66 (2010) 1026-1027.

[4] H. Machida, M. Takesue, R. L. Smith Jr, Green chemical processes with supercritical fluids: Properties, materials, separations and energy, J. Supercrit. Fluids 60 (2011) 2 - 15.

[5] K. Arai, T. Adschiri, Importance of phase equilibria for understanding supercritical fluid environments, Fluid Phase Equilib. 158 (1999) 673-684.

[6] J. M. Kremsner, C. O. Kappe, Microwave-assisted organic synthesis in near-critical water at 300 degrees C - A proof-of-concept study, Eur. J. Org. Chem. 17 (2005) 3672 - 3679.

[7] C. M. Kormos, N. E. Leadbeater, Direct conversion of aryl halides to phenols using hightemperature or near-critical water and microwave heating, Tetrahedron 62 (2006) 4728 4732.

[8] A. C. Metaxas, R. J. Meredith, R.J. Industrial Microwave Heating; Peter Peregrinus Ltd: London, U.K.; 1983.

[9] Anton Paar GmbH. Synthos 3000 (Microwave synthesis platform). 
[10] A. Opalinska, C. Leonelli, W. Lojkowski, R. Pielaszek, E. Grzanka, T. Chudoba, H. Matysiak, T. Wejrzanowski, and K. J. Kurzydlowski, Effect of Pressure on Synthesis of Pr-Doped Zirconia Powders Produced by Microwave-Driven Hydrothermal Reaction, Journal of Nanomaterials, 2006 (2006) 98769.

[11] A. K. Jonscher, Dielectric Relaxation in Solids; Chelsea Dielectrics Press Ltd: London, U.K.; 1983.

${ }^{[12]}$ C. J. F. Böttcher, Theory of Electric Polarisation; Elsevier: Amsterdam, Netherlands; 1952.

[13] A. P. Gregory, R. N. Clarke, A review of RF and microwave techniques for dielectric measurements on polar liquids, IEEE Trans. Dielectr. Electr. Insul. 13 (2006) 727-743.

${ }^{[14]}$ S. Jenkins, T. E. Hodgetts, R. N. Clarke, A. W. Preece, Dielectric measurements on reference liquids using automatic network analyzers and calculable geometries, Meas. Sci. Technol. 1 (1990) 691-702.

[15] A. P. Gregory, R. N. Clarke, Dielectric metrology with coaxial sensors, Meas. Sci. Technol. 18 (2007) 1372-1386.

${ }^{[16]}$ K. Okada, Y. Imashuku, M. Yao, Microwave spectroscopy of supercritical water, J. Chem. Phys. 107 (1997) 9302-9311.

[17] S. B. Lee, R. L. Smith, Jr., H. Inomata, K. Arai, Coaxial probe and apparatus for measuring the dielectric spectra of high pressure liquids and supercritical fluid mixtures, Rev. Sci. Instrum. 71 (2000) 4226-4230.

${ }^{[18]}$ G. A. Dimitrakis, M. George, M. Poliakoff, I. Harrison, J. Robinson, S. Kingman, E. Lester, A. P. Gregory, K. Lees, A system for traceable measurement of the microwave complex permittivity of liquids at high pressures and temperatures, Meas. Sci. Technol. 20 (2009) 045901. 
${ }^{[19]}$ R. Gnanasekaran, D. M. Leitner, Dielectric response and vibrational energy relaxation in photoactive yellow protein: A molecular dynamics simulation study, Chem. Phys. Lett. 516 (2011) 102-105.

${ }^{[20]}$ L. Saiz, E. Guàrdia, J. -À. Padró, Dielectric properties of liquid ethanol. A computer simulation study, J. Chem. Phys. 113 (2000) 2814-2822.

${ }^{[21]}$ E. Guàrdia, J. Martí, Density and temperature effects on the orientational and dielectric properties of supercritical water, Phys. Rev. E, 69 (2004) 011502.

[22] A. Y. Zasetsky, A. S. Lileev, A. K. Lyashchenko, Molecular dynamic simulations of terahertz spectra for water-methanol mixtures, Mol. Phys. 108 (2010) 649-656.

[23] J. Cardona, R. Fartaria, M. B. Sweatman, L. Lue, Molecular dynamics simulations for the prediction of the dielectric spectra of alcohols, glycols and monoethanolamine, Mol. Sim. 42 (2016) 370-390.

${ }^{[24]}$ R. Olmi, M. Bittelli, Can molecular dynamics help in understanding dielectric phenomena?, Meas. Sci. Tech. 28 (2016) 014003.

[25] J. Cardona, M. B. Sweatman and L. Lue, Molecular dynamics investigation of the influence of the hydrogen bond networks in ethanol/water mixtures on dielectric spectra, J. Phys. Chem. B 122 (2018) 1505-1515.

[26] V. M. Anisimov, I. V. Vorobyov, B. Roux, A. D. MacKerell, Jr., Polarizable empirical force field for the primary and secondary alcohol series based on the classical drude model, J. Chem. Theory Comput. 3 (2007) 1927-1946.

${ }^{[27]}$ B. R. Brooks, C. L. Brooks, III, A. D. MacKerell, Jr., L. Nilsson, R. J. Petrella, B. Roux, Y. Won, G. Archontis, C. Bartels, S. Boresch, et al., CHARMM: The Biomolecular Simulation Program, J. Comput. Chem. 30 (2009) 1545-1615. 


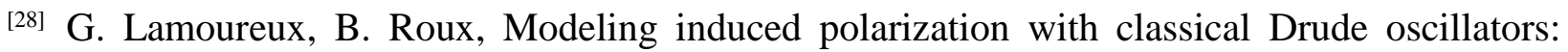
Theory and molecular dynamics simulation algorithm, J. Chem. Phys. 119 (2003) 3025-3039.

${ }^{[29]}$ E. Harder, V. M. Anisimov, I. V. Vorobyov, P. E. M. Lopes, S. Y. Noskov, A. D. MacKerell, Jr., B. Roux, Atomic level anisotropy in the electrostatic modeling of lone pairs for a polarizable force field based on the classical Drude oscillator, J. Chem. Theory Comput. 2 (2006) $1587-1597$.

[30] T. Yamaguchi, C. J. Benmore, A. K. Soper, The structure of subcritical and supercritical methanol by neutron diffraction, empirical potential structure refinement, and spherical harmonic analysis, J. Chem. Phys. 112 (2000) 8976-8987.

[31] A. R. Bazaev, I. M. Abdulagatov, E. A. Bazaev, A. Abdurashidova, PVT measurements for pure ethanol in the near-critical and supercritical regions, Int. J. Thermophys., 28 (2007) 194219.

[32] Y. Hiejima, Y. Kajihara, H. Kohno, M. Yao, Dielectric relaxation measurements on methanol up to the supercritical region, J. Phys.: Condens. Matter 13 (2001) 10307-10320.

${ }^{[33]}$ D. J. Price, C. L. Brooks, A modified TIP3P water potential for simulation with Ewald summation, J. Chem. Phys. 121 (2004) 10096-10103.

[34] Y. Hiejima, M. Yao, Dielectric relaxation of lower alcohols in the whole fluid phase, J. Chem. Phys. 119 (2003) 7931-7942.

[35] T. Yamaguchi, N. Matubayasi, M. Nakahara, NMR study on the reorientational relaxation in supercritical alcohols, J. Phys. Chem. A 108 (2004) 1319-1324.

[36] D. Dellis, M. Chalaris, J. Samios, Pressure and temperature dependence of the hydrogen bonding in supercritical ethanol: A computer simulation study, J. Phys. Chem. B 109 (2005) 18575-18590. 
[37] S. Parez, M. Předota, M. Machesky, Dielectric properties of water at rutile and graphite surfaces: effect of molecular structure, J. Phys. Chem. C 118 (2014) 4818-4834. 